\title{
Evidence for a monosynaptic mechanism in the tonic vibration reflex of the human masseter muscle
}

\author{
E. GODAUX AND JOHN E. DESMEDT
}

From the Brain Research Unit, University of Brussels, Brussels, Belgium

SYNOPSIS Vibration of the masseter and temporalis muscles in normal human adult subjects elicits a tonic vibration reflex with unexpected features. The electromyographic response is not asynchronous as in the limb muscles, but involves well-defined spikes with a one-to-one temporal relation to the vibration cycles. The effect of various parameters such as muscle stretch, vibration frequency or amplitude, etc, has been investigated. The small latency fluctuation of the vibrationinduced spikes is compatible with a monosynaptic reflex mechanism which is considered to be assisted by a polysynaptic facilitatory background of proprioceptive origin.

Current studies of the tonic vibration reflex (TVR) elicited in limb muscles by sinusoidal vibration of the muscle tendon either in man (Eklund and Hagbarth, 1966; de Gail et al., 1966; Rushworth and Young, 1966; Marsden et al., 1969; Hagbarth, 1973; Lance et al., 1973) or in the cat (Matthews, 1966) have emphasized the desynchronized nature and the progressive recruitment of the concomitant electromyographic activities. These features led to the proposal that the TVR involved primarily polysynaptic reflex pathways (Hagbarth, 1973; Lance et al., 1973). The depression of the TVR by barbiturates and other drugs in doses which do not seem to affect the monosynaptic reflexes elicited in the same muscles by a tendon tap (de Gail et al., 1966) appeared to be in line with such an interpretation. Another indirect argument which has been used is the depression of the TVR at levels below a spinal transection in muscles which produce brisk tendon jerks (de Gail et al., 1966). This explanation does not seem to be supported by our finding that jaw vibration in the normal adult man regularly elicited electromyographic (EMG) potentials synchronized with the vibration cycles. These and other results would indeed suggest that the TVR of the masseter muscle involves both a mono-

(Accepted 15 August 1974.) synaptic reflex pathway and a polysynaptic supraspinal facilitatory process.

\section{METHODS}

Sixteen normal unpaid volunteers of both sexes between 20 and 26 years old, were studied. They were free of any symptom or sign of neurological disease. The subjects received no sedation or drug. They were all physically fit and had given informed consent for the procedures, which they subsequently described as rather mild and quite acceptable. A number of subjects were tested several times.

The subjects were seated in an easy chair with the head resting against a sturdy metal frame padded with soft rubber and rigidly fixed on the chair. Two strong elastic bands strapped respectively around the forehead and the mandible (between chin and lower lip) prevented any movement of the head in its receptacle. The fixation was such that the mouth was open with about $5 \mathrm{~mm}$ between upper and lower teeth, thus slightly stretching the masseter and temporalis muscles. The lower elastic band around the chin also served in most experiments to maintain against the mandible a vibrating device of $30 \mathrm{~mm}$ diameter weighing $150 \mathrm{~g}$ which included a compact d.c. motor (12 V maximum). The motor could be fitted easily with any one of 20 different copper loads attached by a screw. When fixed the load had $3.2 \mathrm{~mm}$ eccentricity with respect to the axis of the motor. Unless otherwise specified a load of $16 \mathrm{~g}$ was used in the standard tests. In a few experiments the vibrating device was placed, not on the chin, but 


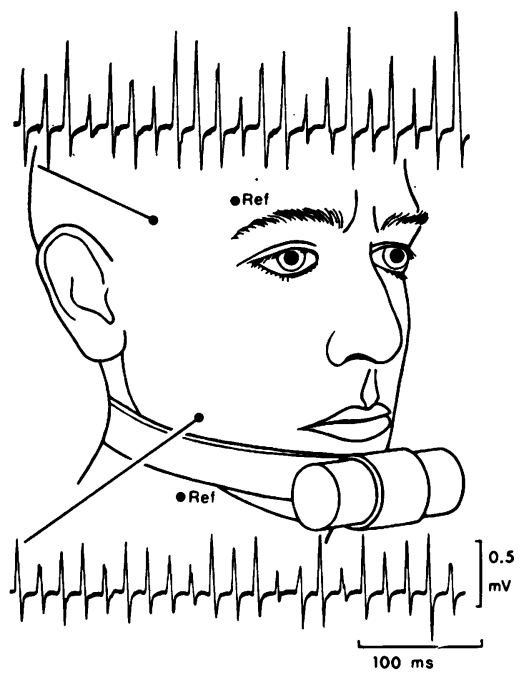

FIG. 1 Sketch of the fixation of the vibrator on the chin and sample electromyograms recorded with cup electrodes on the skin over the temporalis and the masseter muscles during vibration at $57 \mathrm{~Hz}$. The position of the reference electrodes is indicated.

onto the zygomatic bone ipsilateral to the masseter muscle studied. Under the latter conditions the lower elastic band could be dispensed with, in which case the jaw was not maintained in a fixed position with respect to the frame.

The effects of varying the frequency or the amplitude of the vibrations were tested both by fitting the motor with different eccentric loads and by adjusting the d.c. voltages between 2 and $15 \mathrm{~V}$. For any combination of load and current the actual peak to peak amplitude of the vibration with the motor fixed to the jaw was estimated optically with a binocular microscope fitted with a micrometer eyepiece at $\times 15$. When a light was shone on the device, bright lines were seen by reflexion on the vibrator edge. During vibration the lines were expanded into bands whose width, corresponding to the peak-topeak amplitude of mechanical displacement, could easily be estimated. The frequency of vibration was recorded by a silicium semiconductor strain gauge (Philips model PR 9860) interposed between the chin and the motor and displayed on one channel of the cathode ray oscilloscope. When the vibrationinduced muscle responses were to be studied on fast oscilloscope sweeps, the latter were triggered by a built-in system involving a photocell and a beam of light interrupted by each rotation of the eccentric weight on the motor axis. The muscle potentials were amplified by a 10 megohm input impedance preamplifier and displayed on a 565 Tektronix cathode ray oscilloscope using a $3 \mathrm{~A} 9$ plug-in amplifier. The traces were photographed by a Grass C4 camera. The masseter reflex was also studied by percussion of the lower jaw at intervals of five seconds or more (Desmedt, 1973) either with a reflex hammer or with a Racia electro-mechanical hammer rigidly fixed in front of the head frame. The percussion was delivered through a strain gauge device

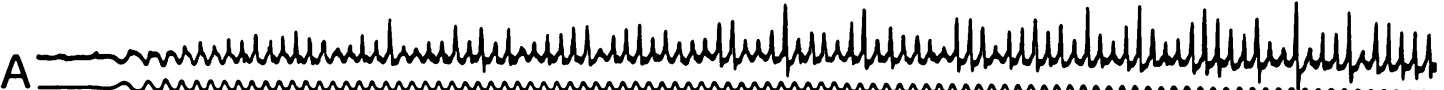
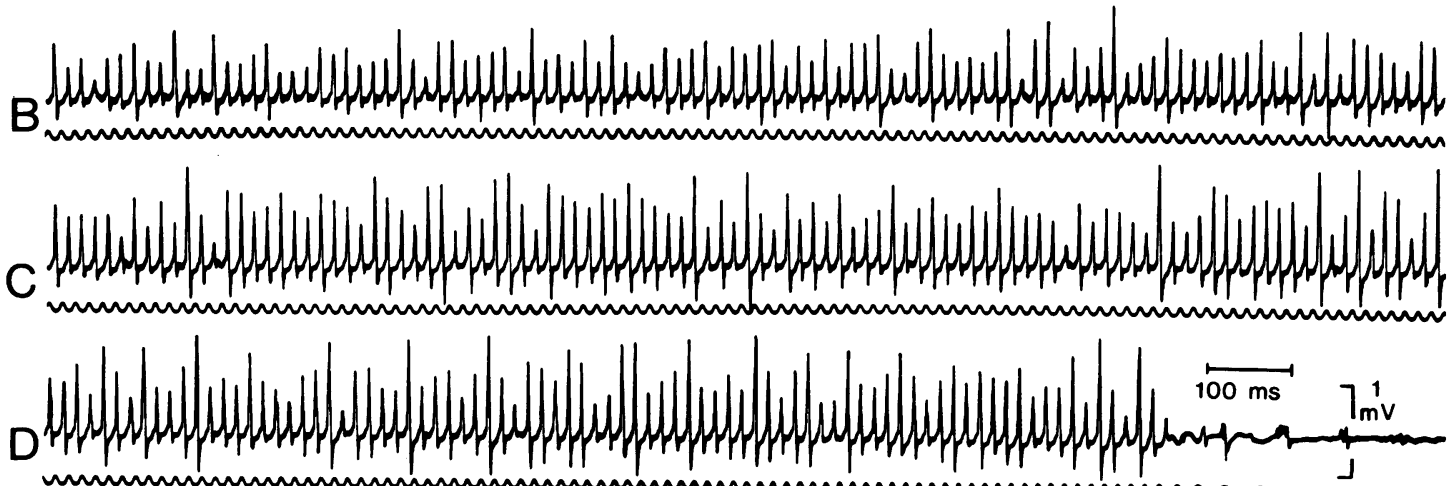

FIG. 2 Continuous recording of a jaw vibration test at $63 \mathrm{~Hz}$ with the masseter electromyogram (upper trace) and the actual vibration recorded by a strain gauge device (lower trace). Vibration amplitude $1.8 \mathrm{~mm}$. The vibrator is switched on at the beginning of $\mathrm{A}$ and switched off at the end of $\mathrm{D}$. Notice the progressive recruitment of the EMG spikes and the briefness of after-discharge. 
placed on the chin which provided the electrical trigger pulse starting the sweep of the oscilloscope.

\section{RESULTS}

Sinusoidal vibration at about $80 \mathrm{~Hz}$ applied to the chin as described above regularly elicited rhythmic electromyographic spike potentials at the vibration frequency in the masseter and temporalis muscles of both sides (Fig. 1). The voltage of 30 consecutive spikes was measured for 10 TVRs in the 16 subjects. The mean voltages varied between 0.5 and $2.5 \mathrm{mV}$. The spike voltage was usually larger in the temporalis muscle. Under steady vibration of the chin the voltage of the successive spikes in any given test varied randomly with no clear indication of spindling, as shown in the various records presented. Slight voluntary contraction of the masticatory muscles increased the size of the vibration-induced spikes without altering this pattern of response.

As regularly observed for TVR of limb muscles (Eklund and Hagbarth, 1966; de Gail et al., 1966) a prolonged phase of gradual recruitment with progressive increase of the electromyographic activity was recorded after the vibration was initiated (Fig. 2). An obvious difference was
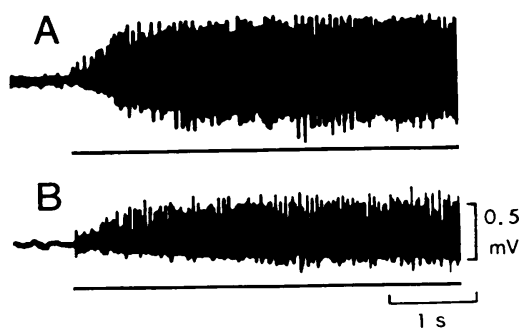

FIG. 3 Vibration test recorded in the temporalis muscle. A: vibrator placed on the chin with a slight stretch of the muscle (cf Fig. 1). B: vibrator placed on the zygomatic bone with the mandible free (isotonic record). The horizontal line below each record corresponds to the vibration.

that in the limb muscles the EMG was asynchronous, whereas in the masseter and temporalis muscles the EMG response to vibration was patterned in spikes at the vibration frequency. When switched on, the motor took about $100 \mathrm{~ms}$ to reach its steady state vibration frequency, as shown by the strain gauge recording on the lower oscilloscope trace (Fig. 2, A). However this does not account solely for the EMG recruitment phenomenon which indeed extended over a period of several seconds. The irregular fluctua-
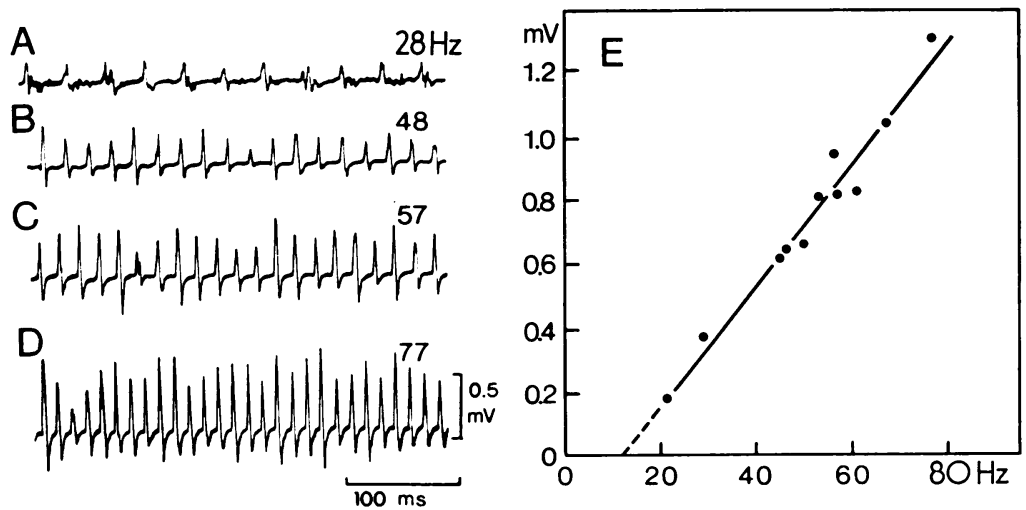

FIG. 4 Effect of different vibration frequencies between 20 and $80 \mathrm{~Hz}$ under constant vibration amplitude at $1.8 \mathrm{~mm}$ in one subject. The vibrator is fixed on the chin and the masseter muscle electromyogram is recorded while the muscle is submitted to a slight constant stretch. A: vibration at $28 \mathrm{~Hz}$. B: vibration at $48 \mathrm{~Hz}$. C: vibration at $57 \mathrm{~Hz}$. D: vibration at $77 \mathrm{~Hz}$. E: diagram with the vibration frequency in each test as abscissa and with the mean voltage of 30 consecutive EMG spikes during steady state vibration as ordinate. The straight line is drawn according to the function calculated (see text). 


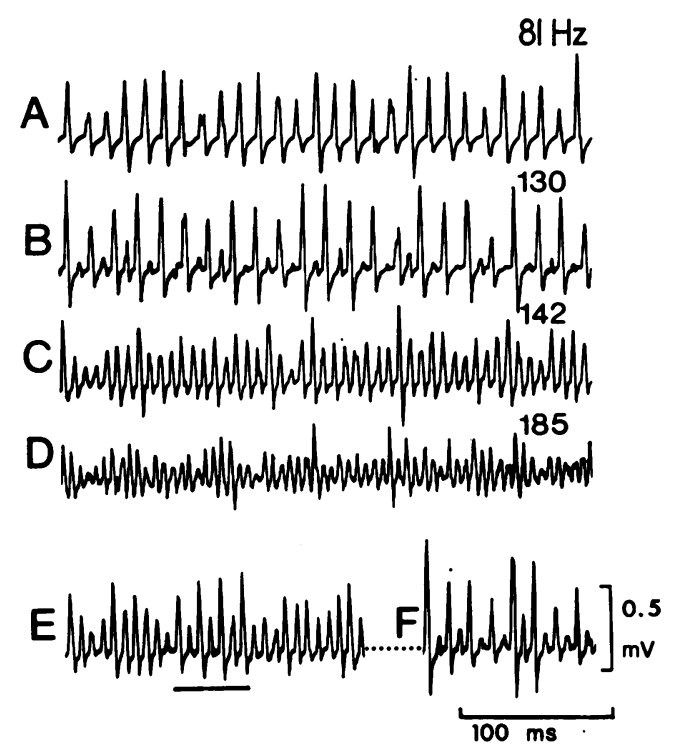

FIG. 5 Same subject as in Fig. 4, tested under identical conditions but with higher vibration frequencies. Masseter muscle electrogram. A: vibration at $81 \mathrm{~Hz}$. B: vibration at $130 \mathrm{~Hz}$ with clear alternating pattern in spike size. C: vibration at $142 \mathrm{~Hz}$ with more regular spike size of lower voltage. $\mathrm{D}$ : vibration at $185 \mathrm{~Hz}$. E, F: another test at $142 \mathrm{~Hz}$ showing sudden transitions between more or less regular and alternating patterns of spike size. tion of the size of the successive vibrationinduced spikes was observed throughout the test. When the motor was switched off the spikes rapidly declined and there was no rhythmic afterdischarge, as if each spike had indeed been related to one vibration cycle (Fig. $2, \mathrm{D})$.

When the vibrator was placed on the zygomatic bone rather than on the chin, it was possible to have the jaw freely hanging with relaxed masticatory muscles. When a zygomatic vibration was initiated under such isotonic conditions rhythmic EMG spikes were initiated in the ipsilateral muscles, the voltage of the spikes being larger in the temporalis muscle than in the masseter muscle. The masseter and temporalis muscles contralateral to the zygomatic vibration remained inactive. The ipsilateral recruiting activity resulted in a progressive closure of the initially relaxed jaw. The mean voltage of $\mathbf{3 0}$ consecutive spikes during steady state remained smaller for the zygomatic vibration (Fig. 3, B) than for the chin vibrations (Fig. 3, A), the latter involving a slight continuous stretching of the masticatory muscles by the rubber band (see Fig. 1) under our experimental conditions.

VIBRATION FREQUENCY Various vibration frequencies between 20 and $200 \mathrm{~Hz}$ were tested to
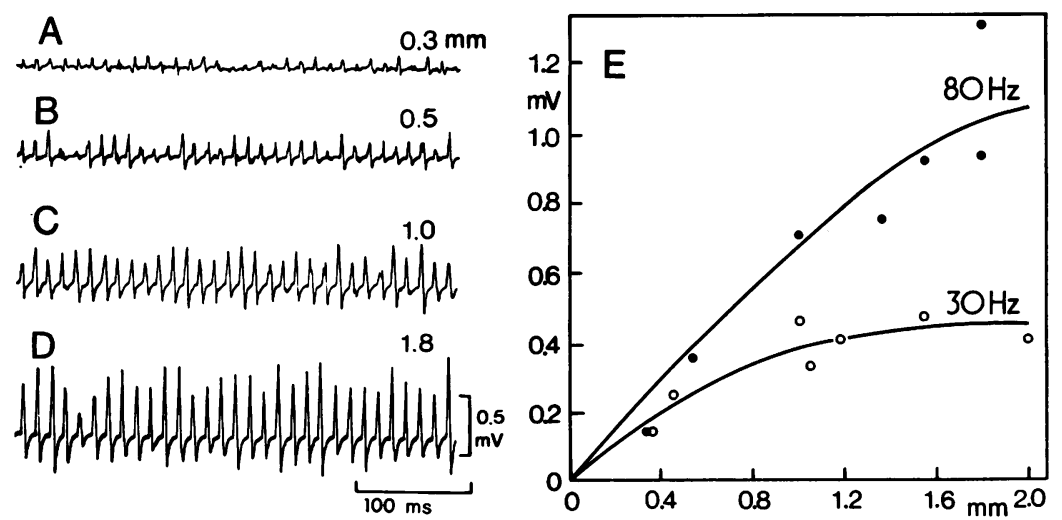

FIG. 6 Effect of different vibration amplitudes at constant vibration frequency. Masseter muscle electrograms recorded under slight constant stretch. Vibrator fixed on the chin. A to D: vibration at $80 \mathrm{~Hz}$ with amplitudes of $0.3 \mathrm{~mm}$ (A), $0.5 \mathrm{~mm}(\mathrm{~B}), 1.0 \mathrm{~mm}(\mathrm{C})$, and $1.8 \mathrm{~mm}$ (D). E: diagram with data obtained in the same subject during two series of tests, one at $80 \mathrm{~Hz}(\mathrm{O})$ and the other at $30 \mathrm{~Hz}$ (O). The vibration amplitudes are plotted as abscissa and the corresponding mean voltage of 30 consecutive EMG spikes during steady state vibration appears on the ordinate. The lines were drawn to fit approximately the experimental points and they were arbitrarily made to pass through the origin. 
analyse the TVR pattern under constant isometric conditions (chin vibration) and no voluntary contraction of the masticatory muscles. The amplitude of the mechanical vibration was measured optically for each frequency with the motor fixed on the subject and it was adjusted to the same chosen value within $5 \%$ by appropriate combinations of the DC voltage and eccentric weight (see Methods).

For frequencies between 20 and $80 \mathrm{~Hz}$ the mean voltage of 30 consecutive spikes during the steady state vibration after the recruitment phase (10 to 30 seconds after starting the motor) increased consistently with the vibration frequency (Fig. 4, A-D). The data for a typical subject are plotted in Fig. 4, E and the calculated regression line was: $y(\mathrm{mV})=0.02 \times-0.21$. The goodness of fit of the regression line was $r^{2}=0.96$.

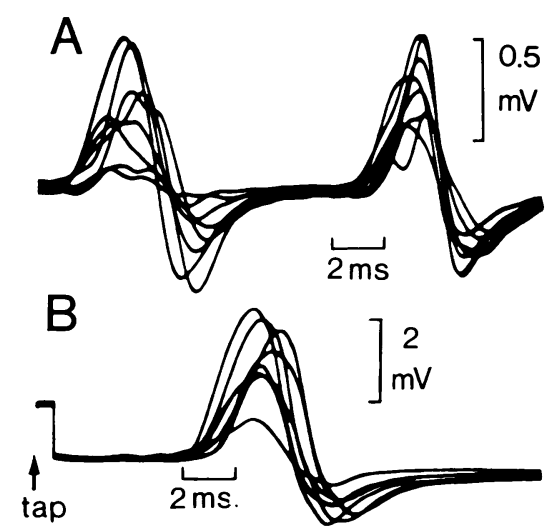

FIG. 7 Comparison of the latency jitter for the masseter EMG spikes during vibration at $88 \mathrm{~Hz}$ with an amplitude of $1.6 \mathrm{~mm}$ (A) and for the masseter reflex (B). The oscilloscope sweep is triggered at a constant angular position of the eccentric weight in the vibrator in $\mathrm{A}$. The vibrator is fixed on the chin and the masseter muscle is kept under slight constant stretch. In $\mathrm{B}$, the sweep is triggered by a strain gauge device excited by the mechanical tap on the chin.

When this straight line is extrapolated downwards it appears that the voltage of the vibrationinduced spikes would become zero for a frequency of $12.5 \mathrm{~Hz}$ in this instance.

On the other hand, for frequencies above about $90 \mathrm{~Hz}$ the mean voltage of the EMG spikes decreased with the frequency (Fig. 5, A-D). At critical frequencies in the range of 110 to $150 \mathrm{~Hz}$ the EMG records showed a clear alternation in the size of the successive spikes, a large spike being followed by a very small spike while the overall frequency of response still corresponded to that of the applied vibration (Fig. 5, B). The alternation pattern sometimes occurred intermittently at some critical frequency in the range considered. In Fig. 5, E, a series of spikes of roughly comparable size changed for a brief episode into a sequence of spikes with a marked alternation in voltage. Later in the same vibration test (Fig. 5, F) a more steady pattern of alternation of larger and smaller spikes was recorded.

VIBRATION AMPLITUDE At any given frequency the amplitude of the applied vibration was tested by using suitable combinations of eccentric weight and DC voltage applied to the motor (see Methods). A range of vibration amplitudes between 0.2 and $2.0 \mathrm{~mm}$ were tested. The mean voltage of 30 consecutive spikes, 10 to 30 seconds after starting the motor, was found to increase with the amplitude of the vibration (Fig. 6, A$D)$. The diagram in $E$ shows the data for two vibration frequencies, 80 and $30 \mathrm{~Hz}$, in the same subject. The mean voltage of the spikes increased up to a maximum value and there was a definite ceiling effect at large vibration amplitudes. On the other hand the maximum voltage was larger at the higher vibration frequencies in the tested range of 30 to $80 \mathrm{~Hz}$.

LATENCY VARIABILITY The rather consistent spike discharges with a one-to-one time relation to the sinusoidal vibration prompted a detailed study of the latency jitter. Each time the eccentric weight on the motor passed a definite angular position in its rotation, an electrical pulse was generated (see Methods) which triggered a fast oscilloscope sweep. The precise time relation of this pulse to the stretching phase of the vibrated masseter muscle was not known but it must have remained constant throughout any steady state vibration test. Figure 7, A shows several sweeps displaying two vibration cycles during such a test: there is a consistent superimposition of the spikes in the successive sweeps in spite of a rather marked voltage variation of the responses. The recorded latency jitter was of the same order 

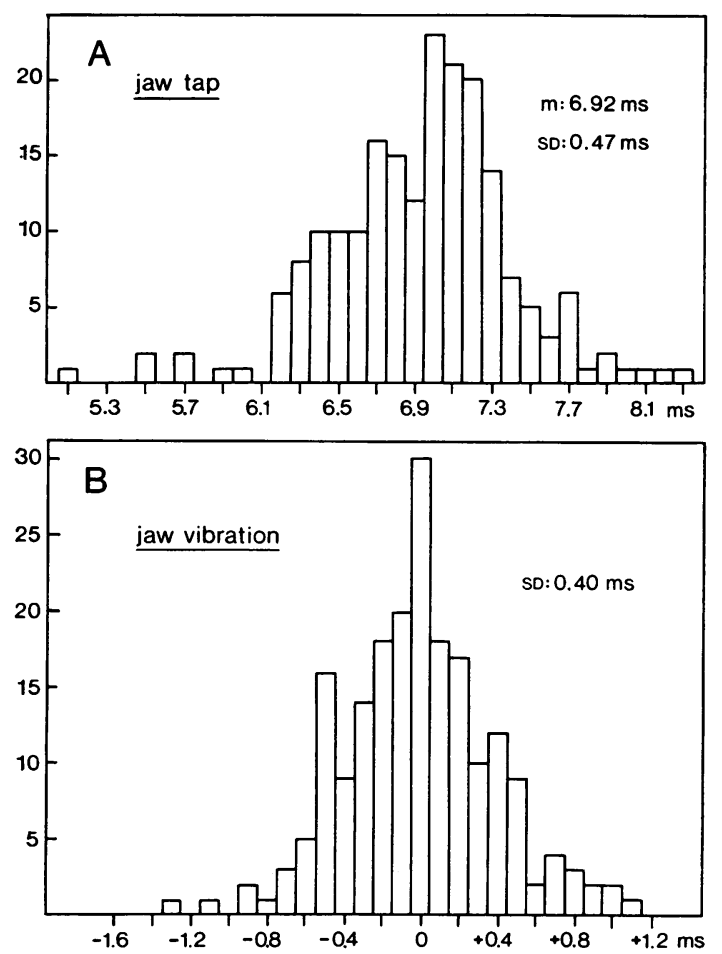

FIG. 8 Diagrams of the latency jitter in the masseter reflex (A) and for vibration-induced EMG spikes (B) in the same subject. Same experiment as in Fig. 7. Two hundred responses are included in each diagram. The abscissa represents the actual reflex latency in $\mathrm{A}$, and the deviation with respect to the modal latency in B (see text).

as that found for the masseter tendon reflex elicited by a mechanical tap on the chin(Fig. 7, B). Figure 8 presents the latency fluctuations for the masseter reflex (A) and for the jaw vibrations at $80 \mathrm{~Hz}(\mathrm{~B})$ on a similar time scale. The mean latency of the masseter reflex was $6.92 \mathrm{~ms}$ in this subject with a SD of $0.47 \mathrm{~ms}$. Similar results were obtained in the other subjects who had mean latencies between 6.8 and $8.5 \mathrm{~ms}$. The latency jitter of the spike elicited by the jaw vibration was plotted relative to a time reference corresponding to the mean latency $(6.1 \mathrm{~ms})$ with respect to the arbitrary but constant time in the vibration cycle. The SD in this case was $0.40 \mathrm{~ms}$. In spite of slight uncertainty about the absolute value of the latency of the vibration-induced spikes, it is obvious that the SD of the latencies were very similar (about $7 \%$ of the mean latency) both for the masseter reflex and for the vibration responses.

\section{DISCUSSION}

It has not been appreciated hitherto that the tonic vibration reflex (TVR) of the masseter and temporalis muscles elicited by sinusoidal vibration of the jaw involves synchronized muscle spike discharges with a one-to-one relation to the vibration cycles (Figs 1,2 ). This pattern of electromyographic response which is not seen for the TVR in human limb muscles under similar conditions (Hagbarth, 1973; Lance et al., 1973) undergoes quite consistent changes when the vibration parameters are modified experimentally.

A well-known feature of the TVR in limb muscles is the progressive recruitment of the asynchronous electromyographic activity (Eklund and Hagbarth, 1966). In the temporalis and masseter muscles such recruitment after onset of a maintained vibration indeed develops over a period of several seconds but it involves $\stackrel{\odot}{\stackrel{\odot}{?}}$

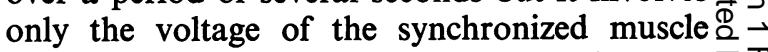
spikes (Fig. 2). Throughout the vibration test $\frac{\mathbb{1}}{0}$ (except for the first few cycles) there is one spike for each vibration cycle. Little after-discharge is recorded when the vibration is discontinued. The rate of recruitment-and indeed the subsequent mean voltage of the muscle spikes during steady state vibration-are increased by a moderate stretch of the muscles. A slight voluntary contraction of the muscles also potentiates the TVR. We did not record the mechanical force produced by the TVR but the pattern of spikes in the TVR was analysed in detail. A linear relation was found between the frequency of jaw vibration between about 20 and $80 \mathrm{~Hz}$ and the mean voltage of the spikes recorded after the recruitment phase during steady state TVR (Fig. 4). Extrapolation of the straight line would suggest that there is a liminal frequency, about $12 \mathrm{~Hz}$ in this example, for eliciting the TVR as indeed found in the cat (Matthews, 1966). At frequencies between about 110 and $140 \mathrm{~Hz}$ the voltage of the spikes tends to alternate as if some process of relative refractoriness were involved. At still higher frequencies there is a decrease in the mean size of the spikes. On the other hand, at any given frequency between about 20 and $80 \mathrm{~Hz}$ the mean 
voltage of the spikes increases with the amplitude of the jaw vibration up to a maximum which depends on the chosen frequency (Fig. 5). For all the parametric sets investigated, and provided the vibration frequency did not exceed about $180 \mathrm{~Hz}$, the muscle spikes maintained a one-to-one relation to the vibration cycle and only the size of the spikes was changed. Random fluctuations of the voltage of successive spikes were observed in all the TVR (Figs 1, 2, 4, 5, and $6)$.

The unusual features of the masseter and temporalis muscles TVR and the remarkable temporal relation between the electromyographic spikes and the vibration cycles raise interesting problems. Experiments on the cat have shown that sinusoidal vibration is a powerful stimulus for spindle afferent nerve fibres and particularly for the large-diameter $I_{a}$ fibres which follow vibration with one spike for each cycle up to frequencies of $400 \mathrm{~Hz}$ (Granit and Henatsch, 1956; Crowe and Matthews, 1964; Matthews, 1966, 1973). Rhythmical oscillations of the membrane potential have been recorded with intracellular microelectrodes in the spinal motoneurones of the cat during vibration of the corresponding muscles (Westbury, 1972). In these animal experiments the motoneurone depolarization was also larger for vibrations of increased amplitude, while static stretch of the vibrated muscle disclosed summation of the depolarizations evoked by both stretch and vibration stimuli (Westbury, 1972). There would seem to be some basis for saying that vibration-induced activity in the large spindle afferent fibres could indeed exert a rather direct effect with preserved temporal patterning onto the membrane potential of the corresponding motoneurones. Along this line an obvious question arising is whether the vibration-induced muscle spikes do have a rather strict temporal relation to the vibration cycles. This was tested by comparing on fast oscilloscope sweeps the latency jitters of the masseter tendon reflex and of the vibration-induced spikes respectively (Fig. 7). The data leave no doubt that, even when the voltage of the vibration spikes fluctuates within a wide range, their latency with respect to an arbitrary point in the vibration cycle remains quite stable. There is in fact little, if any, difference between the latency jitters of the masseter tendon reflex (supposed to be monosynaptic) and of the vibration-induced spikes (Fig. 8). The standard deviation of these latencies is about $0.45 \mathrm{~ms}$ which is only twice the standard deviation of the latency fluctuations of the masseter H-reflex (Godaux and Desmedt, 1975) and of the soleus H-reflex (Trontelj, 1972, 1973). It is indeed not unexpected that monosynaptic reflexes involving the same pathway should have a slightly larger latency jitter when elicited by tendon taps than by direct electrical stimulation of the spindle afferent nerve fibres.

The present evidence is definitely compatible with the suggestion that monosynaptic activation of masseter motoneurones by spindle $I_{a}$ afferent fibres plays a significant role in the TVR. We do not wish to imply that monosynaptic excitation would provide the sole mechanism for the masseter TVR. The slowly progressive recruitment of the electromyographic spikes at the beginning of the TVR (Fig. 2) would suggest that the masseter motoneurones are submitted to an additional facilitatory influence which is more insecure and less straightforward in nature than the monosynaptic excitation. As a working hypothesis it is proposed that a polysynaptic pathway activated by the spindle afferent fibres represents a necessary component of the masseter TVR, in addition to the predominant but insufficient monosynaptic activation. The polysynaptic pathway could presumably be viewed at least partly as supraspinal if the features such as the differential susceptibility to barbiturates and to spinal lesions (as compared with the tendon jerk) shown for the limb muscles TVR (de Gail et al., 1966) were also valid for the masseter TVR. This point must still be left open as we have not yet tested the masseter TVR in this respect.

The proposed model for the masseter TVR thus postulates a major role for the monosynaptic reflex pathway and a superimposed facilitatory effect of a polysynaptic pathway, the temporal patterning of the vibration-induced spikes being related to monosynaptic activation because of the remarkably small latency jitter recorded (Figs 7, 8). We do not think that the processes involved in TVR are fundamentally different in the limb muscles in spite of the asynchrony of the TVR electromyographic response in those muscles. Several recent papers have emphasized that activation of spindle 
afferent fibres in limb muscles by vibration elicits a presynaptic inhibitory effect involving these same afferent fibres, thereby reducing the proprioceptive input from the vibrated muscles (Lance et al., 1968; Gillies et al., 1969; Barnes and Pompeiano, 1970; Burke and Ashley, 1972; Delwaide, 1973; Lance et al., 1973). This inhibitory effect on primary spindle afferent fibres might account for a reduced monosynaptic driving of the limb muscles motoneurones during muscle vibration, thus leaving a major share of the TVR activation in these muscles dependent on the polysynaptic reflex component discussed above. However, as shown in a recent study (Godaux and Desmedt, 1975), the situation is quite different for the masseter muscle whose tendon jerks were shown to be facilitated, not depressed, by jaw vibration. The virtual absence of any depression in the masseter monosynaptic circuit during vibration appears to be related to the lack of reciprocal inhibitory effect arising from jaw opening muscles (Godaux and Desmedt, 1975) and this would obviously allow a stronger monosynaptic excitation of the masseter motoneurones by proprioceptive afferent volleys synchronized with the vibration cycles. Thus the relative contributions of the postulated polysynaptic and monosynaptic mechanisms in eliciting and maintaining the TVR would be biased in favour of the latter mechanism in the case of the masseter and temporalis muscles with their special brain-stem reflex organization.

This research has been supported by the Fonds de la Recherche Scientifique Médicale, the Fonds National de la Recherche Scientifique from which E. Godaux is holding a post-doctoral research fellowship, and the Muscular Dystrophy Associations of America, Inc.

\section{REFERENCES}

Barnes, C. D., and Pompeiano, O. (1970). Presynaptic inhibition of extensor monosynaptic reflex by Ia afferents from flexors. Brain Research, 18, 380-383.

Burke, D., and Ashby, P. (1972). Are spinal 'presynaptic' inhibitory mechanisms suppressed in spasticity? Journal of the Neurological Sciences, 15, 321-326.

Crowe, A., and Matthews, P. B. C. (1964). Further studies of static and dynamic fusimotor fibres. Journal of Physiology, 174, 132-151.

Delwaide, P. J. (1973). Human monosynaptic reflexes and presynaptic inhibition. In New Developments in Electromyography and Clinical Neurophysiology, vol. 3, pp. 508522. Edited by J. E. Desmedt. Karger: Basel.

Desmedt, J. E. (ed.) (1973). Methodology of the triceps surae proprioceptive reflexes. In New Developments in Electromyography and Clinical Neurophysiology, vol. 3, pp. 773780. Karger: Basel.

Eklund, G., and Hagbarth, K.-E. (1966). Normal variability of tonic vibration reflexes in man. Experimental Neurology, $16,80-92$.

de Gail, P., Lance, J. W., and Neilson, P. D. (1966). Differential effects on tonic and phasic reflex mechanisms produced by vibration of muscles in man. Journal of Neurology, Neurosurgery, and Psychiatry, 29, 1-11.

Gillies, J. D., Lance, J. W., Neilson, P. D., and Tassinari, C. A. (1969). Presynaptic inhibition of the monosynaptic reflex by vibration. Journal of Physiology, 205, 329-339.

Godaux, E., and Desmedt, J. E. (1975). Human masseter muscle: H- and tendon reflexes. Their paradoxical potentiation by muscle vibration. Archives of Neurology. (In press.)

Granit, R., and Henatsch, H. D. (1956). Gamma control of dynamic properties of muscle spindles. Journal of Neurophysiology, 19, 356-366.

Hagbarth, K.-E. (1973). The effect of muscle vibration in normal man and in patients with motor disorders. In New Developments in Electromyography and Clinical Neurophysiology, vol. 3, pp. 428-443. Edited by J. E. Desmedto Karger: Basel.

Lance, J. W., Burke, D., and Andrews, C. J. (1973). The reflex effects of muscle vibration. In New Developments in Electromyography and Clinical Neurophysiology, vol. $3 \bar{\sigma}$ T pp. 444-462. Edited by J. E. Desmedt. Karger: Basel.

Lance, J. W., Neilson, P. D., and Tassinari, C. A. (1968)@ Suppression of the $\mathrm{H}$ reflex by peripheral vibration. Pras ceedings of the Australian Association of Neurologists, 5 45-49.

Marsden, C. D., Meadows, J. C., and Hodgson, H. J. F: (1969). Observations on the reflex response to muscle vibration in man and its voluntary control. Brain, 92, 829846.

Matthews, P. B. C. (1966). The reflex excitation of the soleus muscle of the decerebrate cat caused by vibration applied to its tendon. Journal of Physiology, 184, 450-472.

Matthews, P. B. C. (1973). The advances of the last decade of animal experimentation upon muscle spindles. In $\mathrm{New}$ Developments in Electromyography and Clinical Neurophysiology, vol. 3, pp. 95-125. Edited by J. E. Desmedt. Karger: Basel.

Rushworth, G., and Young, R. R. (1966). The effect of vibration on tonic and phasic reflexes in man. Journal of Physiology, 185, 63P-64P.

Trontelj, J. V. (1973). A study of the H-reflex by single fibre EMG. Journal of Neurology, Neurosurgery, and Psychiatry, 36, 951-959.

Trontelj, J. V. (1973). A study of the F response by single fibre electromyography. In New Developments in Electromyography and Clinical Neurophysiology, vol. 3, pp. 318322. Edited by J. E. Desmedt. Karger: Basel.

Westbury, D. R. (1972). A study of stretch and vibration reflexes of the cat by intracellular recording from motoneurones. Journal of Physiology, 226, 37-56. 